\title{
A dynamic new look at the lambda transition
}

\author{
David Goodstein and Andrew R. Chatto ${ }^{\text {a) }}$ \\ Condensed Matter Physics, California Institute of Technology, Pasadena, California 91125
}

(Received 5 November 2002; accepted 2 May 2003)

We discuss aspects of the theory of critical phenomena and explore the superfluid transition in ${ }^{4} \mathrm{He}$. We review some of the recent experimental and theoretical work on helium in nonequilibrium conditions and summarize some future space experiments that might shed light on disagreements between theory and experiment. (C) 2003 American Association of Physics Teachers.

[DOI: $10.1119 / 1.1586263]$

\section{INTRODUCTION}

A phase transition, where one state of matter changes to another, is one of the most beguiling phenomena in the study of matter. The study of phase transitions can reasonably be said to have begun in 1823, when Michael Faraday accidentally liquefied gaseous chlorine. ${ }^{1}$ Faraday was not the first to liquefy a gas, but he was the first to figure out what had happened. The discovery set him off on a long quest to liquefy all the known gases. He would not succeed, but others after him would, leading ultimately to the liquefaction of helium by Heike Kammerling-Onnes in $1908 .^{2}$

Each state of matter exists over a range of temperature and an applied field, such as pressure or magnetic field. Figure 1 shows the regions occupied by the phases of water and helium in the pressure-temperature plane. Each region is bounded by a curve where the phase transition from one state to another occurs. For water, the liquid is bounded at low pressure and high temperature by the vapor pressure curve, where the liquid evaporates, or conversely, the vapor condenses. The low-temperature, high-pressure phase, solid ice, is bounded from the liquid by the melting curve. The three phases coexist at a single, unique point in the $P-T$ plane, known as the triple point.

At first glance, the phase diagram for helium seems to bear certain topological similarities to that of water. It, too, has a vapor pressure curve and a triple point, from which a phase transition rises with a slightly negative slope. However, both the low-temperature phase and the phase transition differ radically from ice and the melting of ice. The low temperature phase is called superfluid helium, and the phase transition is called the lambda transition. Both will be central to the discussion that follows. ${ }^{3}$

Melting and evaporation are examples of first-order phase transitions. The two phases separated by a first-order transition generally differ in both their specific entropy and density. At high temperatures, all vapor pressure curves come to an end at a point where the properties of the liquid and the vapor become indistinguishable. The remarkable Faraday, who called it the disliquefying point, first intuited the existence of this phenomenon. Faraday's successor as Professor at the Royal Institution, Thomas Andrews, gave it its current name in 1869: the critical point. ${ }^{4}$ Although vapor pressure curves always end in critical points, melting curves never do so. They do not because solids differ from liquids in symmetry, a difference that cannot vanish continuously. ${ }^{5}$

\section{THEORY}

\section{A. Critical point phenomena}

Lively interest in critical point phenomena over the past few decades can be traced back to the 1940's, when Guggenheim realized that the gas-liquid coexistence curve is not parabolic, ${ }^{6}$ and Onsager derived an exact solution of the twodimensional Ising Model. ${ }^{7}$ It really picked up steam, however, in the early 1960's when groups in the U.S. and the U.S.S.R. turned their attention to the question of critical point exponents. In the United States, Heller and Benedek investigated the paramagnetic-antiferromagnetic critical transition using nuclear magnetic resonance techniques. ${ }^{8,9}$ In the U.S.S.R., Voronel discovered that the heat capacity of xenon and argon at the gas-liquid critical point becomes infinite. ${ }^{10,11}$ The experimental studies were complemented by theoretical work by Domb, Rushbrooke, Fisher, Marshall, and others. ${ }^{12}$

Critical point phase transitions differ from first-order transitions in that there is no difference in the specific entropy of the two phases at the transition. The phase transitions listed above, as well as the normal fluid-superfluid transition in liquid $\mathrm{He}$, are critical point phase transitions. The heat capacity of liquid ${ }^{4} \mathrm{He}$ at the super-normal transition temperature, the lambda point, is shown in Fig. 2.

To discuss critical point behavior in general, we introduce the notation $t=\left(\left|T_{c}-T\right|\right) / T_{c}$, where $T_{c}$ is the critical point temperature, and $t$ is called the reduced temperature. As a critical point is approached, various properties, such as the heat capacity or the compressibility, go to infinity or zero as power laws in $t$. For example,

$$
C \sim t^{-\alpha},
$$

where $C$ is the heat capacity and $\alpha$ is a critical point exponent. A critical point exponent is defined for each quantity that goes either to zero or to infinity.

Theoretical predictions of relations among the critical point exponents, called scaling laws, and their empirical verification accounted for a good deal of the work that was done in the field of critical point phenomena in the decades after 1960. The essential clue to the physics of critical point phenomena can be found in the well-known phenomenon of critical opalescence. In a common classroom demonstration, a substance such as ethane, which forms a colorless, transparent liquid and gas, is sealed in a strong glass tube at its critical density. At room temperature, the ethane divides into denser liquid and less dense gas, and one easily sees the meniscus between the two states. When it is warmed above its critical point temperature, $32.1{ }^{\circ} \mathrm{C}$, the meniscus vanishes 

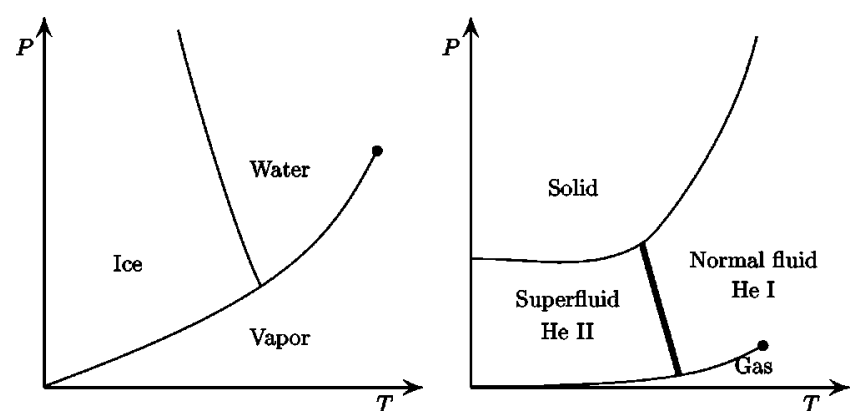

Fig. 1. Phase diagrams in the pressure $(P)$ and temperature $(T)$ plane for $\mathrm{H}_{2} \mathrm{O}$ (left) and ${ }^{4} \mathrm{He}$ (right). Not to scale. Features are exaggerated for topological clarity. Critical points are indicated by a black dot. The line separating superfluid and normal fluid ${ }^{4} \mathrm{He}$ is a line of critical points.

and one sees only a uniform, clear fluid. However, as the fluid cools back toward its critical point, it suddenly becomes completely opaque, the phenomenon known as critical opalescence. As it cools further, striations appear in the opacity, and finally, the meniscus reappears.

Just above the critical point temperature, the state of lowest free energy is a continuous fluid. A bubble of either lower density gas or higher density liquid would have a higher free energy per unit volume by an amount $\delta f$. The probability, $P$, of such a bubble occurring by the usual random fluctuations of statistical mechanics is

$$
P=A e^{-F / k T},
$$

where $A$ is some attempt frequency, $k$ is Boltzmann's constant, and $F$ is the total free energy of the fluctuation. If the volume of the fluctuation is $\ell^{3}$, where $\ell$ is its linear size, then $F=\delta f \ell^{3}$. These fluctuations occur with high probability as long as $F$ is less than $k T$. Therefore, we expect the equilibrium fluid to contain bubbles of liquid and gas on all length scales up to some maximum length $\xi$ such that

$$
\delta f \xi^{3}=k T \text {. }
$$

As $t$ goes to zero, $\delta f$ goes to zero, which means that the length $\xi$ grows to infinity ( $T$ is nearly constant). On the way, $\xi$ becomes as large as the wavelength of visible light. When that happens, light is strongly scattered, and the transparent

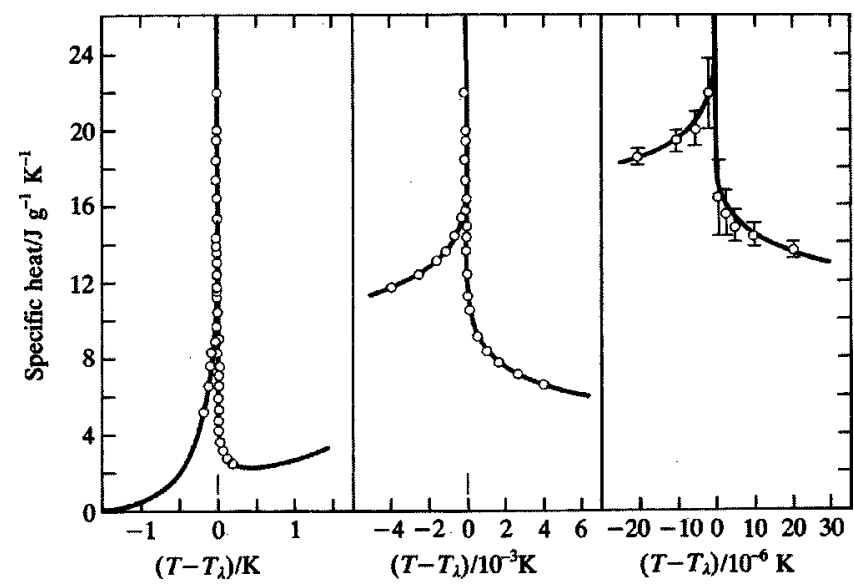

Fig. 2. The specific heat of ${ }^{4} \mathrm{He}$ under saturated vapor pressure as a function of $T-T_{\lambda}$, from a classic paper by Buckingham and Fairbank (Ref. 39). fluid becomes opaque. This is the phenomenon of critical opalescence.

This explanation of critical opalescence contains the essence of the physics of critical point phenomena. As a critical point is approached, correlated fluctuations into the other phase occur on all length scales up to $\xi$, which is known as the correlation length. The correlation length grows to infinity as the critical point is approached, and so it is assigned a critical point exponent $\nu$ :

$$
\xi \sim t^{-\nu} \text {. }
$$

The diverging part of the specific heat is given by

$$
\Delta C=\frac{\partial^{2} \delta f}{\partial T^{2}} \text {. }
$$

According to Eq. (3), with $k T$ constant,

$$
\delta f \sim \xi^{-3} .
$$

We use Eq. (4) to obtain

$$
\delta f \sim t^{3 \nu} .
$$

If we take the second derivative with respect to temperature, the exponent of $t$ is reduced by 2 , and

$$
\Delta C \sim t^{3 \nu-2} \text {. }
$$

But we have already assigned an exponent $-\alpha$ for the heat capacity, so,

$$
\alpha=2-3 \nu \text {. }
$$

Equation (9) is one of the famous scaling laws. If $\alpha$ and $\nu$ can be measured independently, the relation in Eq. (9) can be checked empirically.

\section{B. Critical point phenomena at the lambda transition}

The lambda transition, shown in Fig. 1, is a line of critical points extending from the vapor pressure curve up to the solidification curve at about 25 bar. As shown in Fig. 2, the heat capacity of liquid ${ }^{4} \mathrm{He}$ at the lambda point, and hence the critical point exponent $\alpha$, is among the most carefully measured quantities in all of condensed matter physics. The correlation length cannot be measured directly to test Eq. (9), but there is a very good substitute. Superfluidity is described by a two-fluid model in which the overall density of the fluid, $\rho$, is the sum of two parts, a normal fluid density, $\rho_{n}$, and a superfluid density, $\rho_{s}$. The superfluid density is the part of the fluid that can flow without resistance. At each point in the fluid, the normal fluid and superfluid can have different velocities, $u_{n}$ and $u_{s}$ respectively. The superfluid density goes to zero as the transition is approached from below, with a critical point exponent $\zeta$,

$$
\rho_{s} \sim t^{\zeta} \text {. }
$$

A relatively simple argument ${ }^{13}$ shows that $\zeta=\nu$. Thus for superfluidity, Eq. (9) becomes

$$
\alpha=2-3 \zeta,
$$

where $\alpha$ and $\zeta$ have both been measured ${ }^{14,15}$ and calculated ${ }^{16}$ with great care. The experimental results are

$$
\begin{aligned}
& \alpha=-0.01285 \pm 0.00038, \\
& \zeta=0.6705 \pm 0.0006 .
\end{aligned}
$$




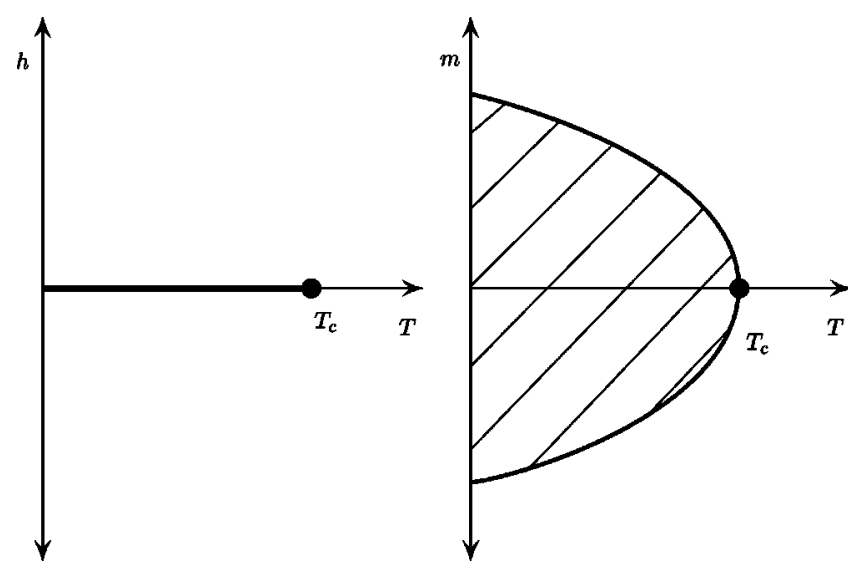

Fig. 3. Generalized phase diagrams in the $h-T$ and $m-T$ planes. Here $h$ is an applied field, $m$ is the system response, and $T$ is the temperature. The bold line on the left and the cross-hatched region on the right represent coexistence of the two phases. $T_{c}$ is the critical temperature.

This result came as something of a surprise. For a long time it was thought that $\alpha$ would be zero (meaning $C \sim \log t$ ) and $\nu$ would be exactly $2 / 3$. Instead, $\alpha$ is negative, meaning the heat capacity does not actually diverge but has a cusp at a finite value. However, Eq. (11) is still obeyed within experimental error.

\section{The external field}

In general, the critical point occurs not only at a certain temperature, but also at a certain value of an externally applied field, such as the pressure (see Fig. 1) or the magnetic field. One can approach the critical point not only by varying the temperature, but also at constant temperature by varying the field, or along a path that varies both temperature and field. Along any such path, the correlation length, $\xi$, goes to infinity as the critical point is approached. If we call the generalized field $h$ and the generalized response of the system $m$, then all points in the $m-t$ plane having the same value of $\xi$ are equivalent. The differential of the free energy density can be written,

$$
d f=-S d T+h d m .
$$

For example, in the gas-liquid transition in Fig. $1, h$ would be the difference between the pressure and the vapor pressure, and $m$ the difference between the density and the critical density. In a magnetic system, $h$ would be the applied magnetic field and $m$ the magnetization. For gas-liquid or magnetic systems, $m$ is known as the order parameter. It appears spontaneously at $h=0$ below the critical point temperature, and goes to zero at the critical point. In both cases it is coupled to the conjugate field, $h$, which can push the system into one of its equilibrium states (gas or liquid, up or down) below the critical point. Generalized phase diagrams in $h, m$, and $T$ are shown in Fig. 3.

It is possible to define a whole new set of critical point exponents. At the critical point, where $(h, t)=(0,0), m$ goes to zero and the generalized susceptibility, $\delta m / \delta h$, goes to infinity. Each gets a critical point exponent. Scaling law relations between these exponents may be found using traditional scaling function arguments, or the same relations may be found simply using Eq. (3) as we have done above.,17 Pressure, which plays the role of conjugate field for the gas-

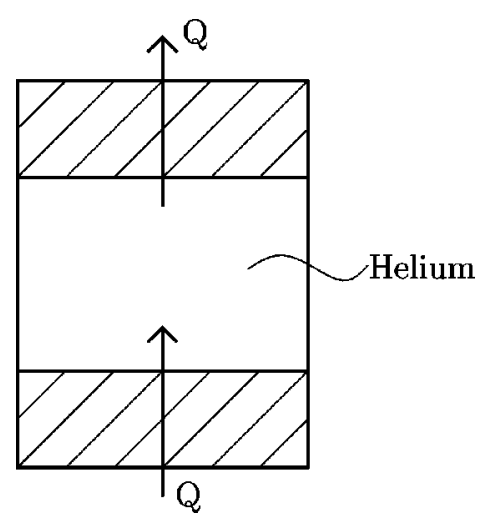

Fig. 4. Schematic experimental configuration. A heat flux $Q$ is injected from the bottom by a heater into a sample of liquid helium. Heat is extracted at the upper end plate, at the same rate $Q$, by means of a thermal network (not shown).

liquid critical point, does not play that role for the lambda transition. As shown in Fig. 1, changing the pressure merely changes the lambda point temperature, but it does not affect any superfluid property. The lambda line in the $P-T$ plane is a line of critical points.

\section{The superfluid order parameter}

The superfluid order parameter is generally taken to be a wave-function-like quantity, $\psi$, given by

$$
\psi=a e^{i \phi},
$$

where $a^{2}$ is proportional to the superfluid density, and the superfluid velocity is proportional to the gradient of the phase, $\phi$. This is a different sort of order parameter than $m$, because rather than having two possible values, its phase can vary continuously from 0 to $2 \pi$ at a given temperature. Presumably, if there existed a field conjugate to $\psi$, it would be able to cause the phase to change from one value to another. No such field exists, at least according to one textbook. ${ }^{18}$ However, we shall argue in what follows that something very much like a conjugate field for superfluidity does exist.

\section{E. A heat flux experiment}

Consider the experiment sketched in Fig. 4. A heat flux, $Q$, is sent into superfluid from below, and exactly the same heat flux is extracted from above. A flux of heat through any ordinary material would produce a temperature gradient, but the superfluid, at least in principle, can conduct heat with no temperature gradient at all. The mechanism by which it conducts heat is called a thermal counterflow, and it is understood using the two-fluid model introduced in Sec. II B. At the lower plate, where the heat is injected, normal fluid is created, and it flows away, carrying the heat. At the upper plate, where heat is extracted, normal fluid is converted to superfluid, which flows back in the other direction, so there is no net flow of mass (at each point, $\rho_{n} u_{n}+\rho_{s} u_{s}=0$ ). Energy can be extracted from the superflow only by creating quantized excitations called phonons and rotons. Because this process entails an energy barrier, there is no dissipation for small counterflow velocities. By virtue of this mechanism, superfluid helium is a superconductor of heat, capable 
of conducting heat without any temperature gradient. According to the two-fluid model, in counterflow close to the lambda point, where $\rho_{n} \cong \rho$, we can write,

$$
Q=-\rho_{s} u_{s} T S,
$$

where $S$ is the entropy per unit mass of the helium. We define

$$
q=\rho_{s} u_{s}=-\frac{Q}{T S} .
$$

Because the quantity $T S$ is essentially constant near the lambda point, $q$ is proportional to the imposed heat flux, $Q$. We will regard $q$ as an imposed external field.

Under most circumstances there are dissipative mechanisms that cause small but measurable temperature gradients to appear in a thermal counterflow. The superfluid part of the helium flows without resistance only below some critical velocity which tends to be very small except in highly restricted geometries. The superfluid also tends to be filled with a tangle of quantized vortex lines that move with the superfluid and interact dissipatively with the normal fluid. (This effect is known as Gorter-Mellink mutual friction. ${ }^{19}$ ) Finally, the normal fluid flow dissipates energy through ordinary viscosity. However, for very small heat fluxes very close to the lambda transition, the super- and normal fluid velocities are given by

$$
\begin{aligned}
& u_{s}=0.8 \times 10^{-2}\left(\frac{Q}{1 \mu \mathrm{W} / \mathrm{cm}^{2}}\right)\left(\frac{10^{-6}}{t}\right)^{\zeta} \mathrm{cm} / \mathrm{s}, \\
& u_{n}=2 \times 10^{-6}\left(\frac{Q}{1 \mu \mathrm{W} / \mathrm{cm}^{2}}\right) \mathrm{cm} / \mathrm{s} .
\end{aligned}
$$

At typical experimental values, $t=10^{-6}$ and $Q$ $=1 \mu \mathrm{W} / \mathrm{cm}^{2}$, the superfluid velocity is less than $0.1 \mathrm{~mm} / \mathrm{s}$, and the normal fluid velocity is nearly four orders of magnitude smaller. Even though we still have $\rho_{n} u_{n}=-\rho_{s} u_{s}$, the fluid is almost all normal, so the normal fluid velocity is very small. Normal fluid viscous heating is negligible, and Gorter-Mellink mutual friction heating, which is proportional to $Q^{3}$, has fallen below the threshold even of the best sub-nanokelvin thermometry, and the superfluid flows without measurable resistance. To an excellent approximation, under these conditions we can take the normal fluid to be at rest in the laboratory frame and the sample to be isothermal. Then the two-fluid model gives for the free energy per unit volume

$$
d f=-S d T+q d u_{s}
$$

where $q d u_{s}$ is the differential kinetic energy density of the superflow, $d\left(\frac{1}{2} \rho_{s} u_{s}^{2}\right)$, if $\rho_{s}$ is independent of $u_{s}$. Compare this expression to the free energy per unit volume of a gasliquid or magnetic critical point,

$$
d f=-S d T+h d m .
$$

We see that $q$ plays the role of the applied field $h$, and $u_{s}$ is the system response to $q$, just as $m$ is the system response to $h$. To be sure, $u_{s}$ is not the real order parameter as $m$ is, but in some ways it can play the same role. For example, we can define a new set of critical point exponents and derive scaling law relations between them. ${ }^{3}$ Let us instead construct phase diagrams for $T, q$, and $u_{s}$ analogous to those for $T, h$, and $m$ in Fig. 3.

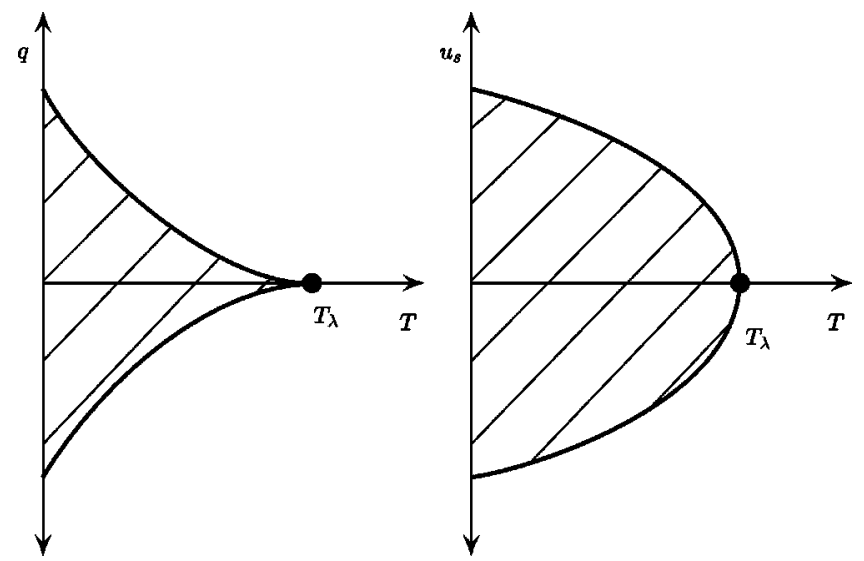

Fig. 5. Superfluid phase diagrams in the $q-T$ and $u_{s}-T$ planes. Superfluidity exists only in the cross-hatched regions. The static lambda transition occurs at $T_{\lambda}$. The curves bounding the cross-hatched regions are $q_{c}(T)$ and $u_{\mathrm{sc}}(T)$.

\section{F. The breakdown of superflow}

Superflow breaks down if the velocity becomes too high. Thus, we can expect superflow to break down at some critical value of the superfluid velocity, $u_{\mathrm{sc}}(t)$, with a corresponding critical value of $q$. This breakdown will be a kind of dynamical phase transition. We can estimate the critical value, $q_{c}(t)$, where superflow breaks down very simply as follows. According to Eq. (3), at $q=0$ the free energy density of the superfluid state close to the lambda transition is lower than that of the normal state by

$$
\delta f=\frac{k T}{\xi^{3}} .
$$

In superconductivity, $\delta f$ is called the condensation energy, and is evaluated from a knowledge of the magnetic field needed to drive the superconductor normal. We can turn the argument around and determine the value of $q$ needed to drive the superfluid normal. That should occur when the condensation energy is balanced by the kinetic energy density of the superflow, $q^{2} / 2 \rho_{s},{ }^{20}$

$$
\frac{k T}{\xi^{3}}=\frac{q_{c}^{2}}{2 \rho_{s}} .
$$

Equation (22) is the equation of a curve in the $q-t$ plane. To approximate it, we use the zero heat current values $\rho_{s}$ $=\rho_{0} t^{\nu}$, with $\rho_{0}=0.34 \mathrm{~g} / \mathrm{cm}^{3}$, and $\xi=\xi_{0} t^{-\nu}$, with $\xi_{0}=3.6$ $\times 10^{-8} \mathrm{~cm} .{ }^{15,21}$ If we express the result in terms of the critical temperature at a given $Q(q=-Q / S T)$, we find

$$
t_{c}(Q)=\left(\frac{Q}{Q_{0}}\right)^{1 / 2 \nu},
$$

where $1 / 2 \nu=0.746$, and $Q_{0} \approx 7000 \mathrm{~W} / \mathrm{cm}^{2}$. Equation (23) implies that $q_{c} \sim t^{2 \nu}$, and $\partial q_{c} / \partial t \sim t^{2 \nu-1}$. Because $2 \nu-1$ is positive, the critical curve approaches the lambda point with zero slope. This result is sketched (for the $q-T$ plane) in Fig. 5.

The corresponding critical velocity, $u_{\mathrm{sc}}$, can be written as $u_{\mathrm{sc}}=q_{c} / \rho_{s}$, and because $\rho_{s} \sim t^{\nu}$, we have $u_{\mathrm{sc}} \sim t^{\nu}$. This curve approaches the lambda point with infinite slope. It is also sketched, in the $u_{s}-T$ plane, in Fig. 5. Compare Fig. 5 
to Fig. 3. In the $h-T$ plane of Fig. 3, coexistence is confined to a single curve. All points not on that curve correspond to possible equilibrium states in which only one uniform phase is present. By contrast, in the $q-T$ plane of Fig. 5, superfluidity, the state where a spontaneous nonzero order parameter exists, is confined to a region, not a single curve. Also, outside of that region, there are no equilibrium points except at $q=0$. A heat flux passing through a nonsuperfluid always produces a temperature gradient, so there can be no state of equilibrium there. The $m-T$ plane resembles the $u_{s}-T$ plane, but the cross hatched regions have somewhat different meanings. In the $m-T$ plane, two phases, liquid and gas or up and down magnetization, coexist in the cross hatched region. In the $u_{s}-T$ plane, the cross hatched region represents a uniform superfluid state, in which the amplitude and the gradient of the phase of the order parameter [Eq. (14)] are determined at each point.

\section{G. Theory}

In 1977, Hohenberg and Halperin classified all possible models, called models A-J, of the dynamics of critical point phase transitions. ${ }^{22}$ The one that is supposed to apply to superfluid helium is called model F. It assumes an order parameter like Eq. (14), and consists of two coupled, nonlinear partial differential equations that, respectively, conserve heat and mass in the flow. These equations cannot be solved in general, but approximate solutions have been offered that yield a good deal of insight into what might be expected. The approximations are either mean-field, ${ }^{23-25}$ which generally gives qualitative insight, or dynamical renormalization group (DRG) calculations to leading order in the coupling constant expansion. ${ }^{26-28}$ The DRG calculations make quantitative predictions that require experimental verification. One result that comes out of the DRG calculations is a prediction of the critical curve along which superfluidity is expected to break down. The prediction is identical to Eq. (23), with exactly the same exponent and very nearly the same amplitude, $Q_{0}$. However, the physics that causes superflow to break down is quite different from the argument that leads to Eq. (23). In the DRG theory, the fact that $\rho_{s}$ depends not only on temperature but also on $u_{s}$ leads to an instability. The reason is quite simple. According to Eq. (19), the superflow contribution to the free energy per unit volume, $\Delta f$, is,

$$
\Delta f=f-f_{0}=\int_{0}^{u_{s}} q d u_{s}^{\prime} .
$$

If $\rho_{s}$ did not depend on $u_{s}$, the integral would give $(1 / 2) \rho_{s} u_{s}^{2}$, so that a plot of $\Delta f$ vs $u_{s}$ would be a parabola. But $\rho_{s}$ is depressed as $u_{s}$ increases, so that the real curve falls below the parabola, as shown in Fig. 6.

According both to mean-field theory and to DRG theory, $\rho_{s}$ is sufficiently depressed to cause the curve to change from convex up to convex down, as shown in Fig. 6. That means there must be an inflection point where the second derivative of $\Delta f$ vanishes. The first derivative, according to Eq. (19) is just $q$. Thus, at the inflection point,

$$
\left(\frac{\partial q}{\partial u_{s}}\right)_{T}=0
$$

In a plot of $q$ vs $u_{s}, q$ rises with increasing $u_{s}$, but reaches a maximum at this point. When this condition is satisfied, the

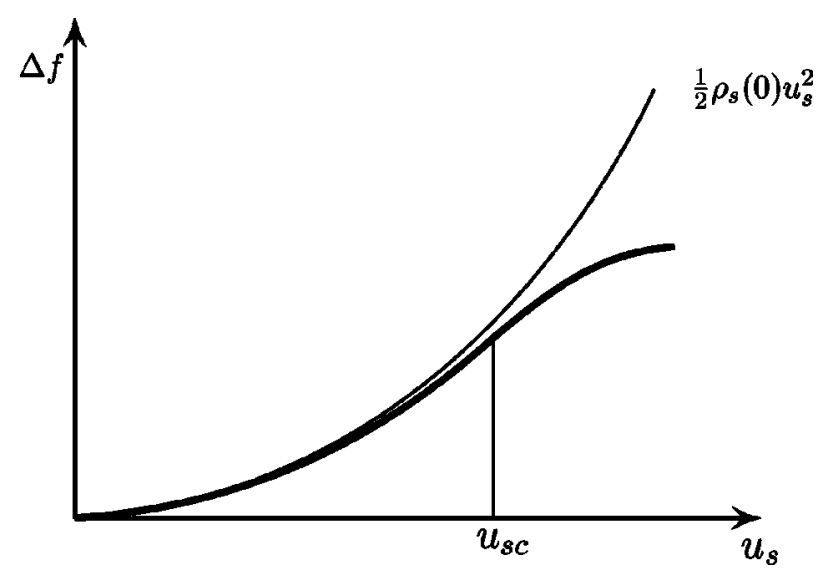

Fig. 6. The excess free energy density vs $u_{s}$. If $\rho_{s}$ were independent of $u_{s}$, the curve would be a simple parabola, indicated by $\frac{1}{2} \rho_{s}(0) u_{s}^{2}$. The real curve, shown in bold, changes from convex up to convex down because $u_{s}$ suppresses $\rho_{s}$. Superflow breaks down at the inflection point.

superflow is unstable. This is perhaps most easily seen by turning the derivative upside down and writing

$$
\left(\frac{\partial u_{s}}{\partial q}\right)_{T}=\infty \text {. }
$$

Thus, uniform superflow is impossible in the presence of even the smallest fluctuations in the imposed $q$ field. Equation (26) is the equivalent of the infinity that occurs in the compressibility along the spinoidal curve of a gas-liquid phase transition. According to Haussmann and Dohm, ${ }^{29}$ whose DRG solution considered fluctuations in the amplitude, but not the phase of the order parameter, Eqs. (25) and (26) are satisfied along the locus of points in the $Q-t$ plane given by Eq. (23), with $Q_{0}=7400 \mathrm{~W} / \mathrm{cm}^{2}$. A later refinement by Haussmann, ${ }^{30}$ which allowed the phase as well as the amplitude to fluctuate, gives the same result, but with $Q_{0}$ $=6600 \mathrm{~W} / \mathrm{cm}^{2}$. We shall refer to the predicted instability temperature as $T_{c}(Q)$.

It is especially interesting that the argument for a true phase transition which led to Eq. (23) and the present argument for a spinoidal instability yield essentially the same result. It is not immediately clear which effect occurs first (or whether they occur simultaneously), and, therefore, whether the breakdown of superflow is actually a line of critical points. If the breakdown of superflow is a line of critical points, we can expect quantities such as the heat capacity to diverge.

Even if the breakdown is a spinoidal instability, the qualitative existence of the inflection point, together with some elementary thermodynamics, gives rise to a divergent heat capacity. Given the new set of conjugate variables in Eq. (19), we can derive the relation between the heat capacity at constant $q$ and at constant $u_{s}$. The derivation is exactly the same as that for the relationship between the heat capacities at constant volume and at constant pressure found in every thermodynamics textbook. The result is

$$
C_{q}=C_{u_{s}}+T V \frac{(\partial q / \partial T)_{u_{s}}^{2}}{\left(\partial q / \partial u_{s}\right)_{T}} .
$$

The heat capacity at constant $u_{s}, C_{u_{s}}$, is what one would measure in a persistent current, where the superfluid is 
trapped in a $u_{s}$ quantum state. According to DRG theory, $C_{u_{s}}$ increases slightly over the heat capacity at rest, $C_{0}$, an effect that is probably too small to be detected experimentally. However, the denominator of the second term on the righthand side of Eq. (27) is precisely the quantity that becomes equal to zero at $T_{c}(Q)$. Thus we can predict on very general grounds that $C_{q}$ should diverge at the critical curve. $C_{q}$ is a quantity that can be and, as we shall see, has been measured in a cell like that shown in Fig. 4.

The reason the heat capacity diverges and superflow is unstable along the critical curve can be understood as follows. The heat capacity is measured by adding heat at constant $q$, where

$$
q=\rho_{s} u_{s}
$$

An increase of the temperature causes $\rho_{s}$ to decrease, so $u_{s}$ must increase. The kinetic energy of the flow,

$$
K \cong \frac{1}{2} q u_{s},
$$

therefore also increases. This increase causes an increased heat capacity,

$$
\Delta C_{q}=\left(\frac{\partial K}{\partial T}\right)_{q}=\frac{1}{2} q\left(\frac{\partial u_{s}}{\partial T}\right)_{q}
$$

But, according to the chain rule for partial derivatives,

$$
\left(\frac{\partial u_{s}}{\partial T}\right)_{q}=-\left(\frac{\partial u_{s}}{\partial q}\right)_{T}\left(\frac{\partial q}{\partial T}\right)_{u_{s}} .
$$

Along the critical curve, $(\partial q / \partial T)_{u_{s}}=u_{s}\left(\partial \rho_{s} / \partial T\right)_{u_{s}}$ and is finite and negative. As we have seen in Eq. (26), $\left(\partial u_{s} / \partial q\right)$ $=\infty$. Thus, $\left(\partial u_{s} / \partial T\right)_{q}=\infty$. That is why the heat capacity is infinite and why ordinary thermodynamic temperature fluctuations break up the superflow along the critical curve, $q_{c}(T)$.

Another prediction that can be drawn from model $\mathrm{F}$ in either the mean-field or the DRG approximation is the temperature distribution in a cell when a normal-fluid/superfluid interface is present. Imagine a cell like that in Fig. 4. In a typical experiment, $Q$ is kept constant while the temperature is made to drift up until superfluidity breaks down at the bottom of the cell. Once the interface has moved into the cell, there is normal fluid at the lower part of the cell. In the normal fluid, $Q$ produces a constant temperature gradient. In the superfluid, the temperature is constant. The region in between is the interface, where the temperature is a nonlinear function of $Q$, as it changes from a constant gradient to a constant as sketched in Fig. 7.

The temperature in the superfluid far from the interface is a unique function of $Q$, which we call $T_{\infty}(Q)$. The relationship between $T_{c}(Q)$ and $T_{\infty}(Q)$ is not known, except, of course, $T_{c}(Q) \geqslant T_{\infty}(Q)$. The width of the interface region is not perfectly well defined, but one can get a sense of what governs it as follows: In the superfluid, or in the normal fluid, the correlation length, $\xi$, grows to infinity as the interface is approached. As the interface is approached from either side, $\xi$ becomes equal to the distance to the (center of the) interface. Beyond that point on either side, $\xi$ is larger than the distance to a boundary, so that there is not effectively an unbounded single phase. This region is the inter-

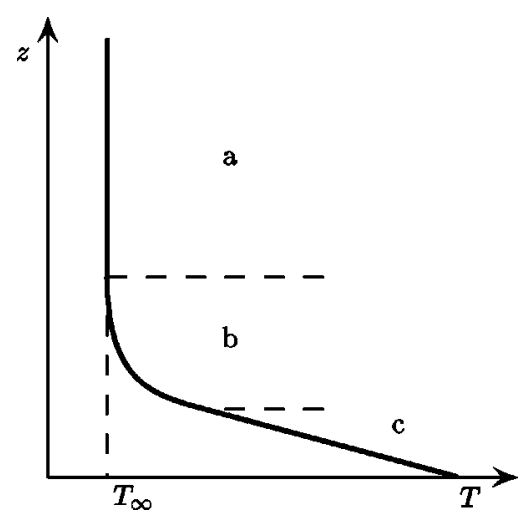

Fig. 7. Temperature profile for helium heated from below. Region (a) is superfluid and has a constant temperature. Region (c) is normal fluid and has a constant temperature gradient. The region that lies between, (b), is the interface.

face. The DRG theory makes predictions of the temperature profile in the interface region. A measurement of the profile would be an important test of the theory.

\section{H. The effect of gravity}

In general, gravity has relatively little effect on bulk superfluidity, because for flow velocities small compared to the speed of sound, the liquid may be regarded as incompressible. However, as seen in Fig. 1, the lambda point temperature decreases with increasing pressure. For this reason the temperature of the lambda transition is lower at the bottom of a cell than at the top because of the weight of the column of helium above it. Other characteristic temperatures, such as $T_{c}(Q)$ and $T_{\infty}(Q)$ are expected to change with height in the cell in the same way as the lambda temperature. Thus, if the cell is all superfluid, the temperature is constant, but the reduced temperature, $t$, is smaller at the bottom of the cell than at the top, and the helium is closer to $T_{c}(Q)$ at the bottom of the cell than it is at the top. The effect is small, the lambda temperature changes by only $1.2 \times 10^{-6} \mathrm{~K} / \mathrm{cm}$ of helium, but at the values of $t$ involved in the phenomena we are discussing here, gravity becomes a crucial impediment to any definitive experimental test of the predictions of theory. To take just one example, the width of the interface region is reduced by gravity to about $200 \mu \mathrm{m}$, too small to be studied in the laboratory. In the absence of gravity, no interface would exist at all without the imposed $Q$. The width of the interface depends on $Q$, and one can imagine using values of $Q$ small enough to make the interface large enough to study.

\section{EXPERIMENT}

Only a few experiments on a superfluid in a heat flux have been reported in the regime where Eq. (19) is a valid approximation. The first, and perhaps the most important of these, was an attempt by Duncan, Ahlers, and Steinberg ${ }^{31}$ to measure $T_{c}(Q)$. They used a cell like the one in Fig. 4. At constant $Q$, they measured the temperature at two different heights in the cell, while the overall temperature was made to drift upward. As long as the cell remained superfluid, the two thermometers tracked closely. But at a certain point the lower one ran away to higher temperatures while the upper one continued to drift at a constant rate (typically, the bath temperature was servoed on the upper thermometer, so its steady 
drift rate was imposed experimentally). The point where the lower thermometer ran away was interpreted as the breakdown of superfluidity, and can be compared to Eq. (23). The result they found did not agree at all with that theoretical prediction. They could express their result in the same form as Eq. (23), but both the amplitude and the exponent disagreed with theory. Instead of $Q_{0}$ equal to 7 or $8 \times 10^{3}$, they found $Q_{0}$ about $600 \mathrm{~W} / \mathrm{cm}^{2}$. And instead of an exponent equal to 0.746 , they found $0.813 \pm 0.012$. The result means that, as one decreases $t$ or increases $q$, superfluidity always breaks down before the theoretical curve is reached. The reason for this discrepancy is not known. We shall refer to this experimental breakdown phenomenon as $T_{\mathrm{DAS}}(Q)$. In an experiment of this kind, once the interface has entered the cell, the upper thermometer is measuring directly the mysterious $T_{\infty}(Q)$. This is less useful than it might seem, however, because of the effect of gravity. In a column of helium in a gravitational field, $T_{\infty}(Q)$ is not constant, but rather depends on where in the cell the interface is. The heat capacity, $C_{q}$ (or, equivalently $C_{Q}$ ) in the superfluid state has also been measured. ${ }^{32}$ To minimize the effect of gravity, the experiment was done in a cell only $0.6 \mathrm{~mm}$ high, so that the difference in reduced temperature from the top to the bottom of the cell was only $1 \times 10^{-7} \mathrm{~K}$. However, excess heat capacity over $C_{0}$ was detected only in the range $t<5 \times 10^{-7} \mathrm{~K}$, so the data must be regarded to be averaged over a range of $t$. Nevertheless, there was an unmistakable discrepancy between theory and experiment. The excess heat capacity was found to be roughly ten times larger than could be accounted for by any theory. For a variety of gravity related reasons, the data were limited to relatively small values of $Q$, far below the breakdown values, $Q\left(T_{c}\right)$ or $Q\left(T_{\mathrm{DAS}}\right)$.

A number of other important experiments have been performed in this area that do not admit to direct comparison to theory, because the theory is not yet capable of predicting their results. One type involves measurements of the thermal conductivity of helium in the normal, interfacial (or nonlinear) and breakdown regimes. ${ }^{33}$ Another is the measurement of the so-called singular Kapitza resistance that occurs when heat passes through a wall bounding liquid helium very close to the lambda transition. ${ }^{34,35}$ There is circumstantial evidence that the DAS phenomenon may actually be caused by the physics of the interface, (not of the bulk helium) of which the singular Kapitza resistance is a symptom. ${ }^{32}$ Finally, there is a class of experiments that actually take advantage of gravity. If, instead of the configuration shown in Fig. 4, heat is put in from above and extracted from below, the column of helium can self-organize into a state of uniform temperature gradient, parallel to the gravitational gradient in the lambda temperature. This is generally referred to as the SOC state (for self-organized critical state). The SOC state was predicted to exist above the lambda transition, maintained by the diverging thermal conductivity at the lambda point. ${ }^{36,37}$ If a bit of the column becomes too cold (that is, too close to the transition), the increased thermal conductivity conducts more heat into it, warming it up, and vice versa. However, the SOC state was observed experimentally both above and below the lambda point temperature, $T_{\lambda} .{ }^{38} \mathrm{~A}$ mean-field solution to model $\mathrm{F}$ indicates that the temperature gradient could be maintained by the dynamical creation of quantized vorticity below the lambda transition. ${ }^{25}$ However, if $t_{c}(Q)$ is a line of true critical points, as suggested above, the thermal conductivity would diverge at this temperature (not at the

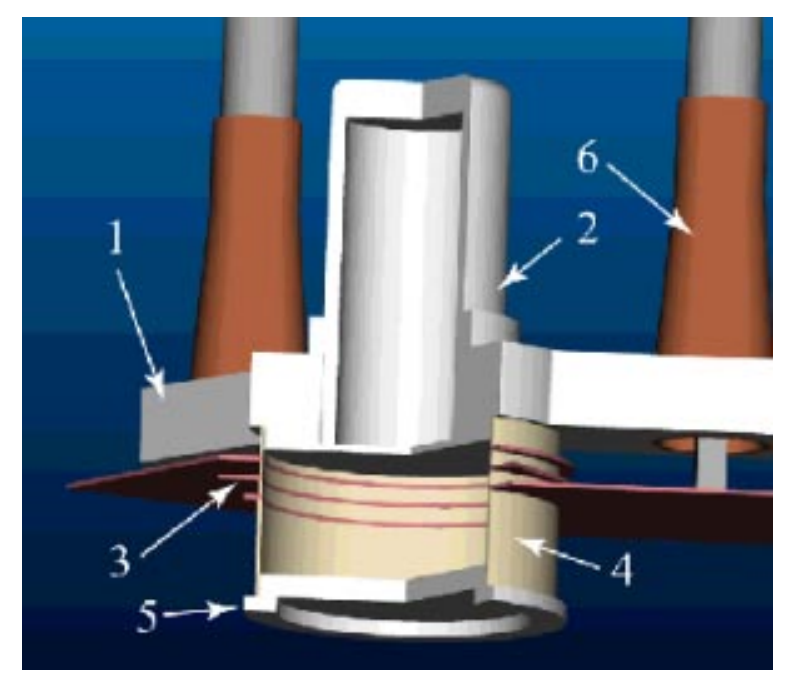

Fig. 8. The DYNAMX cell displayed to scale. The cool plate of the cell (1) supports a bubble chamber (2) where the liquid-vapor interface is maintained. This top endplate also provides structural support for the miniHRTs (miniaturized high resolution thermometers) (6), which are isolated thermally from the top endplate by Vespel standoffs. These miniHRTs attach to their respective sidewall probe copper foils (3), which sample the helium temperature at their height along the thin stainless steel sidewall structure (4). The three sidewall probes are each $1 \mathrm{~mm}$ apart, with the first located 5 $\mathrm{mm}$ from the heated endplate of the cell (5). The third sidewall probe is located $3 \mathrm{~mm}$ below the cool endplate (1).

lambda point), and the argument originally used to predict the SOC state would apply over the entire observed temperature range. ${ }^{38}$ In the SOC state, the temperature is not uniform, but the reduced temperature, $t$, is uniform. A uniform $t$ seems to solve the problem of gravity. However, $t$ is a unique function of $Q$, so it is not possible to use this trick to explore the properties of the whole $t-q$ plane in the laboratory. All possible experiments in this state are restricted to a single curve in the $t-q$ plane.

\section{FUTURE EXPERIMENTS IN SPACE}

In 1992, the Lambda Point Experiment measured the heat capacity of liquid helium at the lambda point in the space shuttle, in the absence of the gravitational pressure gradient and with unprecedented resolution. ${ }^{14}$ Thermometers with sub-nanokelvin resolution, developed for use in that experiment, made possible the entire field of study we are discussing here. A later space-based experiment called $\mathrm{CHeX}$ (Confined Helium Experiment) studied the effect of finite size on the heat capacity at the lambda point. Now a number of new experiments are planned, this time aboard the International Space Station (ISS). Attached to the ISS, the Low Temperature Microgravity Physics Facility (LTMPF) will have a dewar with two experimental cells and a 5-month supply of superfluid helium refrigerant installed and changed out after 6 months or so. The first mission planned for this facility, called M1, will have on board a cell devoted to trying to resolve some of the mysteries we have discussed here. The cell was designed for an experiment called DYNAMX, or DX for short, and will also host a guest experiment called CQ.

A view of the DX cell is shown in Fig. 8. It has three penetrating sidewall thermometers along the helium column. These are thin copper foils braised into the stainless steel 
sidewall, and attached to paramagnetic sensors read out by superconducting quantum interference devices, collectively referred to high resolution thermometers. The basic experiment is to cause the super-normal interface to pass by each of the thermometers at a controlled rate, so that the temperature profile of the interface may be resolved. The CQ experiment will use the same hardware to measure $C_{Q}$ in the absence of gravitational effects. Both experiments will be done over a wide range of $q$ and $t$. Between them they should provide a rigorous test of the DRG solutions of the model $\mathrm{F}$ equations, shed light on the relations between $T_{c}(Q)$, $T_{\infty}(Q)$, and $T_{\mathrm{DAS}}(Q)$, and help resolve the discrepancy between the theoretical and experimental heat capacities.

\section{SUMMARY}

Over the last few decades, advances in theory, including scaling laws and the renormalization group, have led to a dramatic new level of understanding of critical point phase transitions. Among critical point phenomena, the lambda transition in liquid helium has received the most exhaustive experimental attention. Among its many advantages, liquid helium can be made almost perfectly pure chemically, and its remarkable thermal properties make it possible to make measurements of great precision. The most important scaling law prediction, relating the critical point exponents for heat capacity and superfluid density, has been beautifully verified.

In more recent years, the development of high resolution thermometry has made it possible to begin studying the lambda transition under dynamical conditions, by using a heat flux to set up a thermal counterflow in the fluid. Here the situation is strangely different from the satisfying accord between theory and experiment in the case of the static transition. Experiments disagree with straightforward predictions for the critical temperature for the breakdown of superflow, $T_{c}(Q)$, and for the heat capacity at constant heat flux, $C_{Q}$.

Simple predictions and clean experiments can only be made, however, very close to the static lambda point, where the critical heat flux is so small that it does not introduce dissipation and temperature gradients before the superfluid breaks down. Under these very restricted conditions, gravity becomes an important factor. It interferes with the interpretation of data, and it places severe restrictions on the configuration of experiments. For these reasons, it may not be possible to resolve the discrepancies between theory and experiment until experiments can be performed in the absence of gravity.

Fortunately, space beckons. The first round of the necessary experiments is scheduled to be performed in the next few years. DX and CQ and a variety of other experiments will be done in the LTMPF on the ISS, under the sponsorship of NASA. There is every reason to believe that the result will be a new level of clarity and insight into the nature of the lambda transition in particular and of critical point phase transitions in general.

\footnotetext{
${ }^{a}$ Electronic mail: chatto@caltech.edu

${ }^{1}$ D. L. Goodstein, States of Matter (Dover, New York, 1985), p. 266.

${ }^{2}$ H. Kammerling-Onnes, "The Liquefaction of Helium," in Communications from the Physical Laboratory at the University of Leiden (Leiden University, Leiden, 1908), No. 108, p. 18.

${ }^{3}$ P. B. Weichman, A. W. Harter, and D. L. Goodstein, "Colloquium: Criticality and superfluidity in liquid ${ }^{4} \mathrm{He}$ under nonequilibrium conditions," Rev. Mod. Phys. 73, 1-15 (2001). This article provides a more technical approach to some of the same topics as this paper.
}

${ }^{4}$ Reference 1, p. 267.

${ }^{5}$ L. D. Landau and E. M. Lifshitz, Statistical Physics, Part 1 (Pergamon, Oxford, 1980), p. 258.

${ }^{6} \mathrm{E}$. A. Guggenheim, "The principle of corresponding states," J. Chem. Phys. 13, 253-261 (1945).

${ }^{7}$ L. Onsager, "Crystal statistics. I. A two-dimensional model with an orderdisorder transition," Phys. Rev. 65, 117-149 (1944).

${ }^{8} \mathrm{P}$. Heller and G. B. Benedek, "Nuclear magnetic resonance in $\mathrm{MnF}_{2}$ near critical point," Phys. Rev. Lett. 8, 428-432 (1962).

${ }^{9} \mathrm{P}$. Heller and G. B. Benedek, "Nuclear resonance in EuS from $4.2{ }^{\circ} \mathrm{K}$ to the critical temperature region," Phys. Rev. Lett. 14, 71-74 (1965).

${ }^{10} \mathrm{~A}$. V. Voronel, "The heat capacity of Xe near the critical point and the value of Delta-3P,” Zh. Fiz. Khim. 35, 958-959 (1961).

${ }^{11}$ M. I. Bagatski, A. V. Voronel, and V. G. Gusak, "Measurement of the specific heat $C_{v}$ of argon in the immediate vicinity of the critical point," Sov. Phys. JETP 16, 517-518 (1963).

${ }^{12}$ H. E. Stanley, Introduction to Phase Transitions and Critical Phenomena (Oxford University Press, New York, 1971). This book provides a summary of critical point theory up to 1971 .

${ }^{13}$ B. D. Josephson, "Relation between the superfluid density and order parameter for superfluid He near $T_{c}$," Phys. Lett. 21, 608-609 (1966).

${ }^{14}$ J. A. Lipa, D. R. Swanson, J. A. Nissen, T. C. P. Chui, and U. E. Israelsson, "Heat capacity and thermal relaxation of bulk helium very near the lambda point," Phys. Rev. Lett. 76, 944-947 (1996).

${ }^{15}$ L. S. Goldner and G. Ahlers, "Superfluid fraction of ${ }^{4} \mathrm{He}$ very close to $T_{\lambda}$," Phys. Rev. B 45, 13129-13132 (1992).

${ }^{16}$ See M. Campostrini, M. Hasenbusch, A. Pelissetto, P. Rossi, and E. Vicari, "Critical behavior of the three-dimensional $X Y$ universality class," Phys. Rev. B 63, 214503 1-27 (2001), and references therein.

${ }^{17}$ M. E. Fisher, "Scaling, universality, and renormalization group theory," in Critical Phenomena, edited by F. J. W. Hahn (Springer-Verlag, Berlin, 1983).

${ }^{18}$ Reference 1, p. 454.

${ }^{19} \mathrm{C}$. J. Gorter and J. H. Mellink, "On the irreversible processes in liquid helium-II," Physica (Amsterdam) 15, 285-304 (1949).

${ }^{20}$ This form of the kinetic energy density assumes that $\rho_{s}$ is not a function of $u_{s}$. It is actually a lower bound for the kinetic energy at a particular $q$, and therefore implies an upper bound on the calculated $Q_{c}$.

${ }^{21}$ To get $\xi$ from $\rho_{s}$, see Ref. 22, p. 460.

${ }^{22}$ P. C. Hohenberg and B. I. Halperin, "Theory of dynamic critical phenomena," Rev. Mod. Phys. 49, 435-479 (1977).

${ }^{23} \mathrm{~A}$. Onuki, "Theory of helium under heat-flow near the lambda-point. I. Interface of He-I and He-II," J. Low Temp. Phys. 50, 433-454 (1983).

${ }^{24} \mathrm{P}$. B. Weichman, A. Prasad, R. Mukhopadhyay, and J. Miller, "Trapped second sound waves on a nonequilibrium superfluid-normal interface," Phys. Rev. Lett. 80, 4923-4926 (1998).

${ }^{25} \mathrm{P}$. B. Weichman and J. Miller, "Theory of the self-organized critical state in nonequilibrium ${ }^{4} \mathrm{He}$," J. Low Temp. Phys. 119, 155-179 (2000).

${ }^{26} \mathrm{R}$. Haussmann and V. Dohm, "Nonlinear heat-transport near the lambdatransition in ${ }^{4} \mathrm{He}$," Z. Phys. B: Condens. Matter 87, 229-246 (1992).

${ }^{27}$ R. Haussmann and V. Dohm, "Heat-flow induced anomalies in superfluid ${ }^{4}$ He near $T_{\lambda}$," Phys. Rev. Lett. 72, 3060-3063 (1994).

${ }^{28} \mathrm{R}$. Haussmann, "Heat transport and self-organized criticality in liquid ${ }^{4} \mathrm{He}$ close to $T_{\lambda}$," J. Low Temp. Phys. 114, 1-10 (1999).

${ }^{29} \mathrm{R}$. Haussmann and V. Dohm, "Depression of the superfluid transition in ${ }^{4}$ He-renormalization-group theory,” Phys. Rev. B 46, 6361-6373 (1992).

${ }^{30} \mathrm{R}$. Haussmann, "Liquid ${ }^{4} \mathrm{He}$ near the superfluid transition in the presence of a heat current and gravity,” Phys. Rev. B 60, 12349-12372 (1999).

${ }^{31}$ R. V. Duncan, G. Ahlers, and V. Steinberg, "Depression of the superfluid transition-temperature in ${ }^{4} \mathrm{He}$ by a heat current," Phys. Rev. Lett. 60, 1522-1525 (1998).

${ }^{32}$ A. W. Harter, R. A. M. Lee, A. Chatto, X. Wu, T. C. P. Chui, and D. L. Goodstein, "Enhanced heat capacity and a new temperature instability in superfluid ${ }^{4} \mathrm{He}$ in the presence of a constant heat flux near $T_{\lambda}$," Phys. Rev. Lett. 84, 2195-2198 (2000).

${ }^{33}$ R. V. Duncan, A. V. Babkin, D. A. Sergatskov, S. T. P. Boyd, T. D. McCarson, and P. K. Day, "Decoherence under a heat flux near the superfluid transition in ${ }^{4} \mathrm{He}$," J. Low Temp. Phys. 121, 643-652 (2000).

${ }^{34} \mathrm{H}$. Fu, H. Baddar, K. Kuehn, and G. Ahlers, "The boundary resistance between superfluid ${ }^{4} \mathrm{He}$ near $T_{\lambda}$ and a solid surface," Fiz. Nizk. Temp. 24 (2), 101-103 (1998).

${ }^{35}$ K. Kuehn, S. Mehta, H. Fu, E. Genio, D. Murphy, F. Liu, Y. Liu, and G. Ahlers, "Singularity in the thermal boundary resistance between superfluid 
${ }^{4} \mathrm{He}$ and a solid surface," Phys. Rev. Lett. 88 (9), 095702-1-095702-4 (2002).

${ }^{36}$ J. Machta, D. Candela, and R. B. Hallock, "Self-organized criticality in ${ }^{4}$ He with a heat current," Phys. Rev. B 47, 4581-4581 (1993).

${ }^{37}$ G. Ahlers and F. C. Liu, "4He very near $T_{\lambda}$ heated from above," J. Low Temp. Phys. 105, 255-266 (1996).
${ }^{38}$ W. A. Moeur, P. K. Day, F. C. Liu, S. T. P. Boyd, M. J. Adriaans, and R. V. Duncan, "Observation of self-organized criticality near the superfluid transition in ${ }^{4}$ He," Phys. Rev. Lett. 78, 2421-2424 (1997).

${ }^{39}$ M. J. Buckingham and W. M. Fairbank, "The nature of the $\lambda$-transition in liquid helium," in Progress in Low Temperature Physics, edited by C. J. Gorter (North-Holland, Amsterdam, 1961), Vol. 3, pp. 80-112.

\section{OBSERVATIONS OF SCHRÖDINGER'S CAT}

When it comes to atoms, language can only be used as poetry. The poet, too, is not nearly so concerned with describing facts as with creating images.

-Neils Bohr

When I'm in the box, what I hear mostly is the sound of my own body: little rifflings, twisted roars, sometimes a whirr. And smell, I notice thatmy own warm smell I mean-the box is thick, too thick for anything outside to make its way to me.

I can feel, too, the edges of my body, my claws with their uneven points, my hard paws, my little antennae whiskers. I know I am spotted, black and white because I can feel the tiny difference between my colors: black like putty or molasses, white slick as oily paint against my sandy tongue.

There's nothing to do here but play with the devicethat little poison toy that you imagine I ignoreand so I do. I roll it over and over, press my nose against it, even toss it in the air now and again. My fate is randomly controlled. And so I play. I might as well.

Of course I grow hungry and thirsty, but these experiments are brief, I'm out in time for meals. And then I'm at the dish instantly, and you think
I'm not listening as you talk about my life, the way you have created me, the way I'm only here because you witness me,

and when I arch my back and purr, and you stroke me and think I' $m$ ignorant while you are not-I'm laughing at your theories. Really, you have missed it all.

Put this into your formulae: I can see myself in risky darknesses, I am my own witness to my life, I do not live or die because you watch. Put this in too:

sometimes my solitude expands the space between the nucleus and electrons of every atom until I am vast, floating cloudlike over you, watching you go about your other experiments, floating over the ocean like a hurricane, floating out into space, observing everything at the same instant.

And if one day you find me dead in my little box, you will never know what that means, whether I am gone like a snuffed light, or whether i am sill roving among the dim and distant stars.

Patricia Monaghan, Dancing with Chaos (Salmon Publishing Ldt, Claire, Ireland, 2002). 